\title{
Research on the Influence of Celebrity Live Streaming on Chinese Consumers' Purchase Intention
}

\author{
Yirong $\operatorname{Sun}^{1, *}$ \\ ${ }^{1}$ College of Communication, Boston University, Boston, USA \\ *Corresponding author's Email: wendys21@bu.edu
}

\begin{abstract}
The research topic of this paper is the impact of celebrity live broadcast on Chinese consumers' purchase intention. In this paper, 300 questionnaires were randomly distributed by the method of investigation and research. The research shows that celebrity live broadcast is neutral to Chinese consumers' purchase desire, which is basically consistent with the hypothesis. Most people know that the behavior of live consumption is through the recommendation of relatives and friends, not advertising or celebrities; the decisive factor of live broadcast consumption is that the price is lower than other platforms, not the celebrity effect; the public consumption brought by celebrity live broadcasting is not very high overall. This paper reveals the challenges and concerns faced by the streaming media live broadcasting market from the relationship between emerging live broadcasting platforms, celebrity culture and consumer behavior, which has made a specific contribution to the exploration of consumer communication and entertainment media environment and will provide some enlightenment for the development of the industry.
\end{abstract}

Keywords: Celebrity live streaming, Purchase intention, Reputation, Quality.

\section{INTRODUCTION}

A commodity is a kind of good or resource. It has completed or substantial interchangeability as well as economic value or service. With the progress and reform of the social structure, and the increase of people's material needs, people have more choices and financial strength to buy all kinds of goods. With the development of the Internet, live streaming has become a new way of selling goods. More and more individuals, groups, capitals, etc., see the commercial value in it. Among them, celebrities have joined the live-streaming trend increasingly. Among them, the styles of various live-streaming and the retail value presented are different. Live streaming E-Commerce has also become a hot topic in society. Therefore, this research focuses on whether the celebrities' live broadcasts impact the public's consumption. Through the questionnaire survey, this paper hopes to further understand the public's views and attitudes towards star live broadcasting.

\section{LITERATURE REVIEW}

\subsection{The definition of Live Streaming $E$ - Commerce}

Live Streaming E-Commerce refers to a new service method. It uses live broadcast technology to display products at close range, answer consultations, and shopping guides through Internet platforms. In addition, it can be open live broadcast rooms by the stores themselves or promoted by professional anchors or celebrities [1]. Since 2019, major e-commerce platforms have launched live broadcast delivery models. Watching the live broadcast while placing an order without leaving home has become a new choice for many consumers [1].

Users can buy anything daily, choose the author they like to watch, and constantly switch in the live broadcast room to select their favorite products. There are a wide variety of optional products on these live broadcast platforms, which are cheap and convenient. People can more intuitively understand the product's style, purpose, and use through the live broadcast. Moreover, many anchors will introduce the product's advantages and point out the bad points simultaneously. 
Furthermore, the most important factor for shopping in the live broadcast room is the price. The commodities displayed in some live broadcast rooms can often be the lowest on the entire network. In addition, users can receive special coupons, especially in the live broadcast rooms, to obtain benefits.

In addition, the rise of live delivery methods is based on a smooth network, fast payment, and rapid logistics development. The live broadcast itself has a sense of interaction, and the collective participation of many netizens has also brought about the emergence of a herd mentality [1]. Price advantage and the personal charm of the anchor often become the highlight of the live broadcast. The business logic of the boom in live streaming is the conversion of traditional e-commerce to social e-commerce. The way of information transmission is increasingly shifting from graphics to video. At the same time, consumers' shopping habits are changing from traditional shelf search and price comparison to social e-commerce buying methods recommended by acquaintances and opinion leaders [1].

\subsection{The History of Live Streaming $E$ - Commerce in China}

With China's reform and opening in the 1990s, more and more TVs became popular in Chinese families, and TV shopping rose. For example, Beijing TV launched a shopping channel, ranging from health care products for the elderly to learning machines for children to make the most intuitive presentation on TV programs in 1996. The TV shopping industry has reached its peak step by step. In the era of rolling broadcasts on major TV shopping channels across the country, sales exceeded 40 billion yuan. The best of them, such as Hunan Satellite TV's Happy Shopping, contributed 1/3 of revenue on its own and was packaged and listed by Hunan TV, Mango TV, Tianyu Media, and a series of high-quality assets [2]. However, with the "Notice on Strengthening the Management of TV Shopping Short Film Ads and Home Shopping Programs" in 2010, live TV shopping started to go downhill. However, with the advent of the social e-commerce era, from the mid-to-late 10 s to the early 20s, especially live shopping based on Taobao and Douyin, it has begun to reach the period where everything can be live streaming [2].

With the epidemic at the end of 2019, the Chinese stayed at home and turned live e-commerce broadcasts into a part of their lives [2]. Almost all commercial forms are migrating to live streaming, and nearly all celebrities, internet celebrities, and entrepreneurs have come to join this national show. Today, Internet giants are entering live broadcasting. It is difficult to estimate the specific number of practitioners in the e-commerce live broadcast industry. However, there is a new situation with Taobao, Douyin, and Kuaishou as the leader, which is staging. According to data from the
Foresight Research Institute, as of March 2020, the number of live webcast users has reached 560 million, accounting for $62.0 \%$ of all netizens. Among them, the number of e-commerce live broadcast users is 265 million, and the penetration rate is as high as $29.3 \%$ [2].

\subsection{Live Streaming E-Commerce in Other Countries}

Live e-commerce seems to have become a battleground for e-commerce and social giants. In the other countries, at present, the market is currently being divided by two forces. One force is the on-site store broadcast model (such as Amazon and Shopee), and the format is almost the same as Chinese e-commerce platforms [3]. The other force is the online celebrity live broadcast model represented by social media, such as TikTok, YouTube, Instagram, and Facebook [3].

YouTube: YouTube announced that it would conduct a "grayscale test" of live shopping in its app. It reports that this function is only available to a few creators and brands, and it may be open to more creators later this year. As a video platform with more than 2 billion monthly livelihoods, YouTube and its creators are aware of the platform's robust business. As the "interest" in e-commerce gradually heats up, YouTube is also constantly developing functions, which is better for the needs of creators [3].

Amazon: With the rise of social media live broadcasts, at the end of 2019, Amazon announced the launch of live broadcast functions on the entire site and launched an app similar to Taobao's live broadcastAmazon Live Creator-to attract more influencers and brands to adopt the live streaming mode [3].

Facebook \& Instagram: With the launch of the store function, Facebook is also testing the feasibility of live shopping in its software on a small scale. Facebook finally opened the live shopping function to the public, and at the same time, launched a series of activities called "Live Shopping Friday" [3].

However, from the current point of view, live streaming on platforms in other countries is more inclined towards traditional product introduction and evaluation. Consumers in other countries usually pursue brand exposure rather than selling goods, while Internet celebrities in other countries define themselves more as "creators," not "sellers" [3].

\subsection{The Definition and Influence of Celebrity Effect}

The Celebrity effect is the effect of attracting attention, strengthening things, and expanding influence achieved by the appearance of celebrities. Nowadays, the celebrity effect has had a profound impact on all aspects of people's real life [4]. For example, celebrity 
endorsement advertisements can stimulate consumption, and celebrity participation in charitable activities can drive social care for the weak, and so on. In other words, the celebrity effect is equivalent to a brand effect, and its impact can be as powerful as a crazy star chaser [4]. Before the Internet age, people's understanding of celebrities may be limited to film and television stars, singers, political figures, news anchors, etc. However, in this Internet age, the definition and scope of celebrities are changing.

With the rapid development of social networks, related topics-living streaming-have become more and more topics of concern. According to the literature review in the previous article, the definition of Live Streaming E-Commerce, the history of E-commerce Live broadcast in China, the development status of ecommerce live broadcasting in other countries, and the explanation of celebrity effect, this research will analyze the impact of celebrity live broadcasts on consumers.

\section{METHODS}

The hypothesis of this study is that the influence of celebrity live broadcast on Chinese consumers ' purchase intention is neutral, neither increase nor decrease.

To continue to explore this hypothesis, this paper conducted a background investigation and research on the idea, worked on a research design, and obtained specific data. Our research type is a survey, which is a descriptive study and a cross-sectional study. This study requires all participants to have at least understood or purchased celebrity live streaming. The purpose is to measure the results of the research accurately.

In this study, a total of 300 participants filled out an anonymous quantitative questionnaire. To facilitate the collection of questionnaires and allow participants to be familiar with the questions, the online questionnaire of this study conducted through the "Questionnaire Star" of the WeChat platform (a Chinese social application). The questionnaire was randomly distributed in WeChat Moments or Group Chats, ensuring that the collected data has a certain degree of objectivity and sample diversity. There are 20 questions in the questionnaire, including views and attitudes towards the live streaming of celebrities. The questionnaire sets up easy-to-answer and straightforward questions, such as yes-no questions, multiple-choice questions (divided into single-choice and multiple-choice), and subjective questions (choose a number from 1 to 10 to indicate the degree). In this way, it is better to collect relevant ratio and preference data. In addition, several of the problems are collecting basic personal information, such as gender, education, experience strength, etc. It takes about 5 minutes to complete the entire questionnaire. At the end of the questionnaire, it states that the data of the questionnaire will be fully recorded for further transcription and data analysis, and it will not be disclosed to anyone other than the researcher. Next, I will elaborate on the data after setting up a questionnaire to demonstrate that Chinese consumers' buying intentions will not be influenced by celebrity live streaming.

\section{RESULTS}

\subsection{Result 1}

We asked the public from which channel to know about the content and programs related to celebrity live streaming. I gave four choices, and people can only choose one. The four options are: recommendations by friends and relatives, advertising (including online and offline), self-initiated click-in, and others. The figure 1 shows that in the 300 questionnaires, 198 people chose recommendations from relatives and friends, accounting for $66.0 \%$ of the total, 68 people chose advertising pushes, accounting for $22.7 \%$, and 28 people chose to actively click into the live broadcast, accounting for 9.3\%. Six people are choosing other channels, accounting for $2.0 \%$. It objectively shows that the recommendation of relatives and friends is when people learn about the celebrity live broadcast and enter the live broadcast room. The proportion of advertising recommendations is not very large for people to understand the live celebrity broadcast.

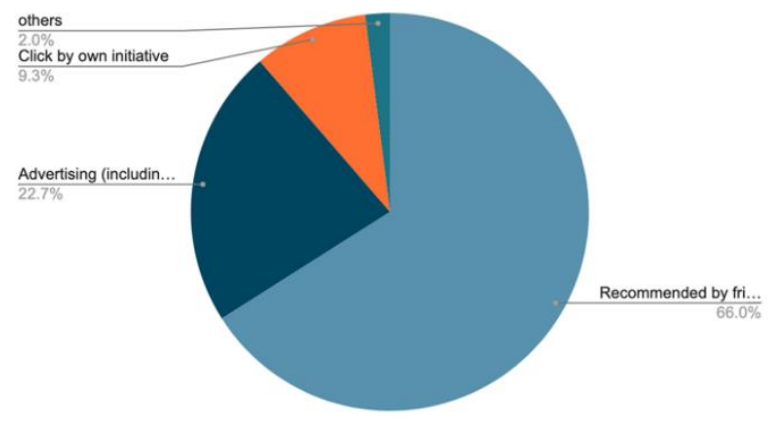

Figure 1 Channels for the public to know and enter the celebrity live

\subsection{Result 2}

We asked about the consumption of watching celebrity live streaming in the past three months. We have given three options, and the respondent can only choose one option for this question. The first option is less than 100 yuan, the second one is 100-1000 yuan, and the third one is more than 1,000 yuan. There are a total of 300 responses. Among them, 210 people choose below 100-yuan, accounting for $70.0 \%, 74$ people choose $100-1,000$-yuan, accounting for $24.7 \%$, and 16 people choose more than 1,000-yuan, accounting for $5.3 \%$. This data shows that most people spend relatively small amounts when watching live celebrity broadcasts. 
However, a small number of people still pay a relatively large amount of money after watching live celebrity broadcasts.

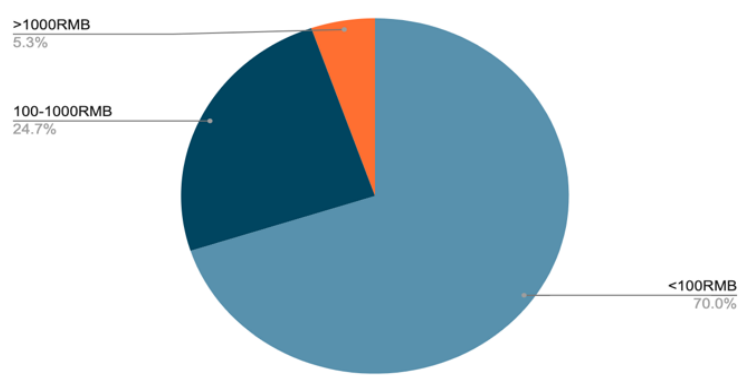

Figure 2 Consumption data of watching celebrity live streaming

\subsection{Result 3}

We asked, what directly caused your consumption in the celebrity live broadcast room? We have given six options, and the respondent can only choose one option. The first option is celebrity recommendation. The second option is for personal needs. The third option is a recommendation by friends and relatives. The fourth is the temptation of price (cheap). The fifth is advertising recommendation. The sixth is the other.

There are a total of 300 answers. The first option is 52 people, accounting for $17.3 \%$, and the second option is 47 people, accounting for $15.7 \%$, the third option is 41 people, accounting for $13.7 \%$, the fourth option is 120 people, accounting for $40.0 \%$, the fifth option is 39 people, accounting for $13.0 \%$, and the sixth option is one person, accounting for $0.3 \%$.

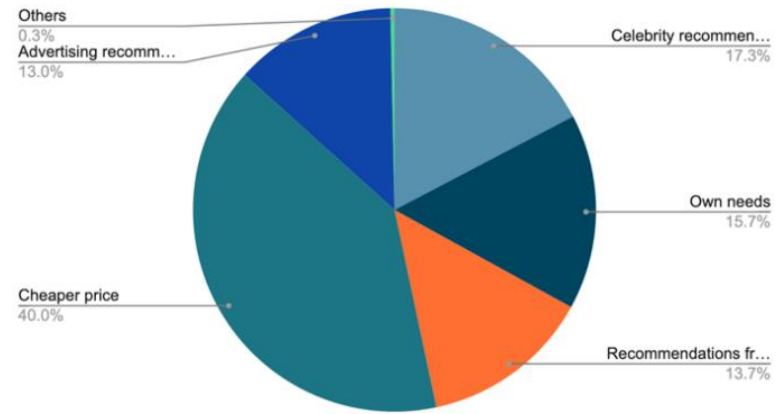

Figure 3 Main factors of public consumption in celebrity live streaming room

According to these statistics, the majority of people's consumption behavior after watching celebrity live streaming is primarily due to lower prices than on other channels. In contrast, other factors' ratio is almost the same (recommendations from celebrities, family and friends, advertising, and a person's own needs). It shows that these are not decisive factors in promoting consumer behavior, and the effect is similar.

\section{DISCUSSIONS}

The above data survey reflects the current public attitudes and views on celebrity live broadcasting. Although the commercial data of star live broadcasting is excellent, many problems still test and affect the public's confidence and attitude towards star live broadcasting.

First, the sales data in the star live broadcast rooms is inaccurate, and there are fraudulent behaviors. Although there was a live broadcast debut of Huang Zitao (huge fan base Chinese star) some time ago, his unpretentious and authentic live broadcast style made him declare no script and other grounding actions. The first show achieved a dazzling result of 230 million GMV [5]. The live broadcast lasted 11 hours, and the total number of orders exceeded 1.6 million, of which the single product sales exceeded 74,600 [5]. However, compared with last year's 2020, this year's star live streaming has gradually cooled down. It is not ideal for the data of a large number of celebrities streaming live. Not everyone can convert reputation into sales. For example, a particular brand paid 800,000 to Li Xiang (a famous Chinese host), but she did not sell a single item during the five-minute live broadcast; Xiao Shenyang (a famous comedian) sold liquor [6]. There were more than 20 orders in the live broadcast room, but 16 orders returned immediately afterward [6].

Second, there is a problem with the quality of goods in the live streaming of celebrities. Problems with live streaming products are generally due to loopholes in product selection, brand authorization, and management specifications of the live streaming team. The second reason is that the anchor team is tempted by commercial interests to take risks and know that fakes are sold. This situation is terrible and suspected of violating laws and regulations [7]. Then, the product problem will undoubtedly directly damage the rights of consumers.

Third, in the beginning, by the celebrity's reputation, live streaming can attract people. But, if live streaming wants to retain customers for a long time, it must rely on integrity and quality. In addition, many consumers already know that the routines of celebrity live broadcasts (brainwashing yells, panic countdowns, and atmosphere rendering) and will not buy goods easily. For example, Zhang Chenguang, an old drama player, cried tears and apologized to fans in the live broadcast, making the public feel that it was self-acted commercial marketing [8].

Mainly due to the previous three reasons, the public did not want to waste time watching celebrity live streaming. They consider why they should watch live broadcasts when they buy something. They may be involved in the age of consumption. At the same time, because of the low price, the celebrity's reputation, the recommendation of relatives and friends, many people 
continuously enter the live streaming to purchase something [9].

Therefore, the influence of celebrity live streaming on Chinese consumers' purchase intention is neutral, neither increasing nor decreasing. In the future, some solutions can solve the problems existing in the current star live broadcast room. For example, the set of advertising and other relevant laws can restrict the behavior of live streaming. The products cannot be exaggerated or false. For another example, MCN organizations, e-commerce companies, celebrity studios, brand owners, etc. can reach cooperation and carry out a reasonable and effective live broadcast mechanism instead of blindly, unrestricted, and irregular live streaming behaviors [10].

\section{CONCLUSION}

This research paper focuses on the impact of celebrity live streaming on Chinese consumers' purchase intention. First, starting from background research, it explores the definition of live streaming, the development of live streaming in China, the status of live streaming in other countries, and the purpose and influence of celebrity effects. Then, based on the quantitative questionnaire, the following findings can be roughly drawn. 1) Most people understand that the live broadcast is through the recommendation of relatives and friends rather than advertising marketing or celebrities; 2) At present, the amount of public consumption in the celebrity live streaming is not very high, but there are some occasional high examples; 3 ) In the live broadcast room, the decisive factor for mass consumption is lower prices than on other platforms, rather than celebrities or advertising recommendations.

In addition, this research has made a specific contribution to the exploration of consumer communication and the entertainment media environment. The collected data and findings provide insights for the analysis of human consumption behavior. This research also reveals the challenges and concerns facing the live streaming market, from the relationship between emerging live broadcast platforms, celebrity culture, and consumer behavior, which will provide some enlightenment for the development of the industry.

\section{REFERENCES}

[1] Why is live streaming so popular, Xinhuanet, 30 April. 2020, http://www.xinhuanet.com/local/202004/30/c_1125925405.htm/. Accessed 30 Oct. 2021.

[2] Live delivery: from 1992 to 2020, Jiemian, 10 Nov.2020,https://www.jiemian.com/article/524825 1.html/. Accessed 30 Oct. 2021.
[3] Giants' E-commerce Anxiety: Overseas Live Streaming Brings, Jiemian, 26 July. 2021, https://www.jiemian.com/article/6405147.html/. Accessed 30 Oct. 2021.

[4] Xuean $\mathrm{Wu}$, Internet celebrities should deliver positive energy, Chinese Financial News, 26 Sept. 2014,

https://www.financialnews.com.cn/wh_138/ly/201 409/t20140926_63493.html/. Accessed 30 Oct. 2021.

[5] Yutong $\mathrm{Hu}$, The first show with goods sold 230 million, Huang Zitao is the new "ceiling" in the live broadcast industry, Jiemian, 26 Oct. 2021, https://m.jiemian.com/article/6746281.html/.

Accessed 30 Oct. 2021.

[6] Celebrity Live Streaming is to "fever": data fraud, Thepaper, 12 March, 2021, https://www.thepaper.cn/newsDetail_forward_116 77028/. Accessed 30 Oct. 2021.

[7] Lei Li, Why does the celebrity live streaming's farce continue, NBD, 23 Junly. 2021, http://www.nbd.com.cn/articles/2021-0713/1840702.html/. Accessed 30 Oct. 2021.

[8] Vanity Fair Live Broadcasting Disillusionment: Is the celebrity's live broadcast no longer effective, Finiance Sina, 27 June, 2021, https://finance.sina.com.cn/tech/2021-06-27/docikqciyzk2072981.shtml/. Accessed 30 Oct. 2021.

[9] Yulei Chen, Is it so magical to the Internet celebrity live streaming. Finiance Sina, 7 April, 2020, http://finance.sina.cn/zl/2020-04-07/zliimxyqwa5462190.d.html?from=wap/. Accessed 31 Oct. 2021.

[10] Understand MCN and live streaming in one article, Xueqiu, $\quad 8$ Jan. 2020 , https://xueqiu.com/3936065506/138935278/. Accessed 31 Oct. 2021. 